\title{
Daily Melatonin Levels in Women with Systemic Lupus Erythematosus
}

\author{
Mehmet Ağıll11, İbrahim Aydın², Fevzi Nuri Aydın² \\ ${ }^{1}$ Department of Biochemistry, Ağrı Military Hospital, Ağrı, Turkey \\ ${ }^{2}$ Department of Biochemistry, Sarıkamış Military Hospital, Sarıkamış, Kars, Turkey \\ ${ }^{3}$ Department of Biochemistry, Şırnak Military Hospital, Şırnak, Turkey
}

Dear Editor,

Sir, in a recent issue of Balkan Medical Journal, we have read with a great interest the published article by Robeva et al. (1) entitled "Decreased daily melatonin levels in women with systemic lupus erythematosus - a short report". The authors showed that daily melatonin levels were decreased in women with systemic lupus erythematosus (SLE). However, we think that some points should be discussed.

Melatonin is a hormone that found in plants, animals and microorganisms. Melatonin is mainly produced and secreted by pineal gland in humans. Secretion occurs in dark, and peaks at 2 to 4 a.m. (2). Its production is suppressed by exposure of light to retina. It is especially blue light, at a wavelength of around 460 to $480 \mathrm{~nm}$, which inhibits melatonin secretion in the morning. $90 \%$ of the hormone is cleared from circulation in one passage through the liver after secretion. Because of these causes, serum samples obtained at after dawn are not suitable for evaluating melatonin levels. 6-hydroxymelatonin sulphate (6-HMS) is the major hepatic metabolite of melatonin and is primarily excreted in the urine. It has been accepted that urine 6-HMS is a good biomarker of pineal gland activity for desired period (3).

Several kinds of plants contain considerable amount of melatonin such as banana, orange and pineapple. Therefore, consuming such foods give rise to significant increment on serum melatonin levels (4). In this study, the authors did not define the eating habits of participants in terms of above plants. In addition, for the control group, psychiatric disorders, medication (e.g. $\beta$-blockers), and metabolic disorders should be expressed in detail.

The authors have stated that the mean age was not significantly different between the two groups. However, they com- pared three groups: short photoperiod SLE, long photoperiod SLE and control groups. It is known that melatonin production clearly declines with age (2). In this regard, the mean ages of three groups should be defined clearly, to assess whether there were differences and to prevent misunderstanding.

The diagnosis of SLE is based on a combination of clinical findings and laboratory evidence which have been defined by the American College of Rheumatology (ACR). The ACR recommends antinuclear antibody (ANA) testing in patients with two or more unexplained signs or symptoms of SLE. According to the Evidence-Based Guidelines for the use of ANA testing, the average sensitivity of the ANA was $93 \%$ and the specificity was $57 \%$ for the diagnosis of SLE (5). In this regard, the rate of ANA positivity in patients with SLE in this study, which was $71 \%$, is questionable.

In conclusion, as we believe that this study contributes valuable information to the literature, an explanation of above concerns will certainly present reliable data to the readers.

Author contributions: Concept - M.A.; Design - M.A.; Supervision - İ.A.; Data Collection\&/or Processing - F.N.A.; Analysis\&/or Interpretation - M.A., İ.A.; Literature Search - M.A., F.N.A., İ.A.; Writing - M.A.; Critical Reviews - F.N.A.

Conflict of Interest: The authors declare that they have no conflict of interest.

\section{REFERENCES}

1. Robeva R, Tanev D, Kirilov G, Stoycheva M, Tomova A, Kumanov P, et al. Decreased Daily Melatonin Levels in Women with Systemic Lupus Erythematosus-A Short Report. Balkan Med J 2013;30:273-6. 
2. Pandi-Perumal SR, Srinivasan V, Maestroni G, Cardinali D, Poeggeler B, Hardeland R. Melatonin. FEBS J 2006;273:281338. [CrossRef]

3. Ozkan E, Yaman H, Cakir E, Deniz O, Oztosun M, Gumus S, et al. Plasma melatonin and urinary 6-hydroxymelatonin levels in patients with pulmonary tuberculosis. Inflammation. 2012;35:1429-34. [CrossRef]

4. Sae-Teaw M, Johns J, Johns NP, Subongkot S. Serum melatonin levels and antioxidant capacities after consumption of pineapple, orange, or banana by healthy male volunteers. J Pineal Res 2013;55:58-64. [CrossRef]

5. Solomon DH, Kavanaugh AJ, Schur PH; American College of Rheumatology Ad Hoc Committee on Immunologic Testing Guidelines. Evidence-based guidelines for the use of immunologic tests: Antinuclear antibody testing. Arthritis Rheum 2002;47:434-4. [CrossRef]

\section{AUTHORS' REPLY}

Ralitsa Robeva

Clinical Center of Endocrinology and Gerontology, Medical University Sofia, Sofia, Bulgaria

\section{Dear Editor,}

We are very happy to find that our paper has provoked scientific discussion and we would like to provide some methodological explanations in order to clarify the aforementioned points.

The age of the lupus patients included in the study did not differ according to the photoperiod $(42.07 \pm 11.76$ vs. $41.58 \pm 12.62$ years, $\mathrm{p}=0.840$ ). The patients and controls, whose blood samples were collected in the short photoperiod, were on similar age $(43.83 \pm 12.29$ vs. $41.58 \pm 12.62, p=0.412)$. The controls were clinically healthy people without any known (including psychiatric) diseases. No one reported use of beta blockers. The diet habits between patients and controls were not compared, but the blood samples were collected after an overnight fast in both groups. Moreover, we have no reasons to believe that Bulgarian lupus patients consume less exotic fruits than controls.
ANA results could vary widely depending on immunohistochemical methods used for detection and different ANA positivity status was reported in different studies as it could be clearly seen on table 1 of the cited above ref. 5 (1). The patients in our study were recruited consecutively and we did not found reasons to exclude ANA negative patients since the ANA positivity had not been accepted as a mandatory criterion for the disease (2). Nevertheless, we did analyze separately the data of the ANA positive patients and the results were similar. The melatonin levels were lower in patients than in controls $(17.71 \pm 6.92$ vs. $21.63 \pm 6.60 \mathrm{pg} / \mathrm{mL}, \mathrm{p}=0.020)$ and they were inversely correlated to the SLEDAI index in SLE women $(\mathrm{r}=-0.235, \mathrm{p}=0.037)$.

The arguments for the daily melatonin measurement were described in the discussion part of the paper (3). However, we could not explain the reasons for the observed changes in the daily melatonin secretion. They could be related to the chronic disease, chronic immunosuppressive treatment, progressive brain vasculitis, lupus disease per se, or other unknown reasons. Therefore, we strongly believe that the evaluation of the serum melatonin rhythm in different time points during the day and night (instead of urine derivates) in newly diagnosed untreated lupus patients would be of great clinical importance.

Undoubtedly, further studies are needed to assess the importance of the pineal and extrapineal melatonin secretion in systemic autoimmune diseases.

\footnotetext{
Address for Correspondence: Ralitsa Robeva, Clinical Center of Endocrinology and Gerontology, Medical University Sofia, Sofia, Bulgaria

Phone: +3598956042 e-mail: rali_robeva@yahoo.com
}

\section{REFERENCES}

1. Solomon DH, Kavanaugh AJ, Schur PH; American College of Rheumatology Ad Hoc Committee on Immunologic Testing Guidelines. Evidencebased guidelines for the use of immunologic tests: Antinuclear antibody testing. Arthritis Care \& Research 2002;47:434-4. [CrossRef]

2. Cervera R, Espinosa G, D'Cruz D. Systemic lupus erythematosus: pathogenesis, clinical manifestations and diagnosis. In: Bijlsma JW, editor. EULAR Compendium on Rheumatic Diseases. BMJ Publishing Group and European League Against Rheumatism 2009,p.257-68.

3. Robeva R, Tanev D, Kirilov G, Stoycheva M, Tomova A, Kumanov P, et al. Decreased daily melatonin levels in women with systemic lupus erythematosus - a short report. Balkan Med J 2013;30:273-6. 